PoS $\quad \begin{aligned} & \text { PROCEEDINGS } \\ & \text { OF SCIENCE }\end{aligned}$

\title{
Charmonium-like particles at Belle
}

\section{Sookyung $\mathrm{CHOI}^{* \dagger}$}

Gyeongsang National University

E-mail: schoi@gsnu.ac.kr

We report updated results on charmonium and charmoniumlike states from Belle. These include a new state observed in $\gamma \gamma \rightarrow \omega J / \psi$ process and a reanalysis of the $Z^{+}(4430)$ state.

European Physical Society Europhysics Conference on High Energy Physics

July 16-22, 2009

Krakow, Poland

* Speaker.

${ }^{\dagger}$ on behalf of the Belle Collaboration 


\section{Introduction}

A number of new particles have been observed by the B-factory experiments. Some of them do not fit into the predicted charmonium states and, as a result, are candidates for new types of particles such as a hybrid $c \bar{c}$-gluon state or a multiquark state of the molecular type ( $c \bar{q} \bar{c} q$ ) or the diquarkdiantiquark $(c q \overline{c q})$ type. Here, we report a new charmoniumlike state observed in $\omega J / \psi$ final states produced via two photon collisions and discuss the possibility that it may be one of the three states previously reported near $3940 \mathrm{MeV}$. We also report results on the charged charmoniumlike state, the $Z^{+}(4430)$, that are based on a reanalysis using a Dalitz plot formalism. This new analysis confirms previous measurements within their errors. If this is a meson, its minimal quark structure would have to be a $c \bar{c} u \bar{d}$ tetraquark arrangement. In addition, we report updated results on $X(3872)$ and $1^{--} Y$ states produced by ISR together with their counter part in $b \bar{b}(s \bar{s})$ sector.

\section{New Charmoniumlike enhancement in $\gamma \gamma \rightarrow \omega J / \psi$}

Belle observed three states near $3940 \mathrm{MeV}$ in three different decay channels [1][2][3] shown in Fig. 1. Among these three, the $Z(3930)$ state (Fig. 1 c)) produced in the $\gamma \gamma \rightarrow D \bar{D}$ process is generally considered to be the charmonium $\chi_{c 2}^{\prime}$ state, even though the mass $(M=3929 \pm 5 \pm 2$ $\mathrm{MeV}$ ) is somewhat lower than potential model predictions. Figure 1 a) shows the $X(3940)$ in the $D \bar{D}^{*}$ mass spectrum from double charmonium production in $e^{+} e^{-} \rightarrow J / \psi D^{*} \bar{D}$ annihilation. Figure $1 \mathrm{~b}$ ) shows the $Y(3940)$ in the $\omega J / \psi$ mass spectrum in $B \rightarrow Y K$ decays. The mass and width of the $X(3940)(Y(3940))$ are measured to be $\mathrm{M}=3942_{-6}^{+7} \pm 6(\mathrm{M}=3943 \pm 11 \pm 13) \mathrm{MeV}$ and $\Gamma=37_{-15}^{+26} \pm 8(\Gamma=87 \pm 22 \pm 26) \mathrm{MeV}$. Although there is similarity in these masses, the $X(3940)$ and $Y$ (3940) appear to be different states: the $X(3940)(Y(3940))$ has not been seen in the $\omega J / \psi\left(D \bar{D}^{*}\right)$ mass system in $B \rightarrow X(Y) K$ decays. It is important to search for $X Y \rightarrow \omega J / \psi($ or $\left.D \bar{D}^{*}\right)$ in two photon processes, where its spin-parity of resonance is preferentially constrained to be $J^{p}=0^{+}$or $2^{+}$.

Figure 2 shows the $\mathrm{W}$ distribution for candidate $\gamma \gamma \rightarrow \omega J / \psi$ events [4]. Total transverse momentum balance $\left(\left|\Sigma P_{t}\right|\right)$ of final state particles was required to be less than $0.1 \mathrm{GeV}$ in $e^{+} e^{-} \mathrm{CM}$ system. An S-wave Breit-Wigner fit to this enhancement gives preliminary results for the mass and width of $\mathrm{M}=3914 \pm 4 \pm 2 \mathrm{MeV}$ and $\Gamma=28 \pm 12_{-8}^{+2} \mathrm{MeV}$. This signal significance is $7.7 \sigma$. This state, denoted by $X(3915)$, is probably related to one of the three above-mentioned states in this mass region. If we assume the $X(3915)$ is $0^{+}\left(2^{+}\right)$resonance, the product of the two-photon decay width and branching fraction for $\omega J / \psi$ is determined to be $\Gamma_{\gamma \gamma}(X(3915)) \mathrm{B}(\mathrm{X} \rightarrow \omega \mathrm{J} / \psi)=$ $69 \pm 16_{-17}^{+7}\left(21 \pm 4_{-5}^{+2}\right)$ eV for $J^{p}=0^{+}\left(2^{+}\right)$. Meanwhile the product of the two-photon decay width and branching fraction for $Z(3930) \rightarrow D \bar{D}$ is $180 \pm 50 \pm 30 \mathrm{eV}$. For the case of the $X(3915)$ being the $Z(3930)\left(\chi_{c 2} \prime\right)$, the ratio of branching fractions $\frac{\operatorname{BF}\left(\chi_{c_{2}}^{\prime} \rightarrow \omega \mathrm{J} / \psi\right)}{\mathrm{BF}\left(\chi_{\mathrm{c} 2}^{\prime} \rightarrow \mathrm{D} \overline{\mathrm{D}}\right)} \geq 0.08$ is huge for an above-opencharm-threshhold charmonium state. The $\Gamma(\omega J / \psi)$ is also too high to be charmonium which is expected to be the order of $\mathrm{keV}$. If the $X(3915)$ is the same state as both the $Y(3914)$ observed in BaBar and $Y(3940)$ in Belle, the $J^{P C}$ should be $0^{++}$.

\section{Charged resonancelike states}

In 2007 Belle reported the first charged charmoniumlike state, denoted by $Z^{+}(4430)$, in the 

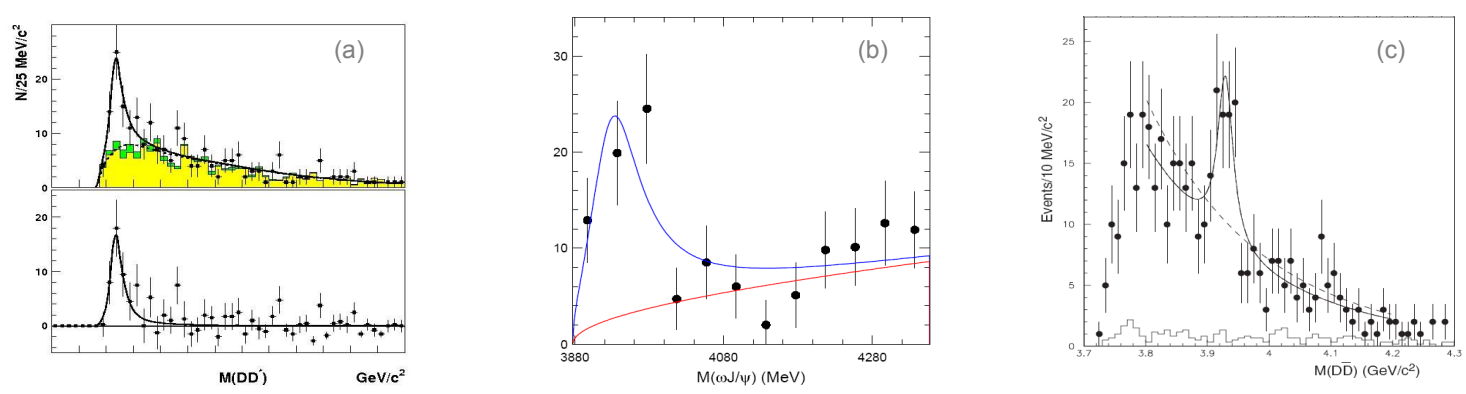

Figure 1: Three states observed in the mass range of 3.90 - $3.95 \mathrm{GeV}$. a) The $M_{X(3940)}\left(D \bar{D}^{*}\right)$ distribution for events produced in $e^{+} e^{-} \rightarrow J / \psi D \bar{D}^{*}$. The solid lines represent the fit results, the dashed lines background. b) $M_{Y(3940)}(\omega J / \psi)$ in $B \rightarrow K \omega J / \psi$ decays and c) $M_{Z(3930)}(D \bar{D})$ in $\gamma \gamma \rightarrow D \bar{D}$ process.

$\pi^{+} \psi^{\prime}$ mass in $B^{0} \rightarrow K^{-} Z^{+}$decays[5]. After vetoing the $K^{*}(892)$ and $K^{*}(1430)$ regions, the mass and width were measured to be $\mathrm{M}=4433 \pm 4 \pm 2 \mathrm{MeV}$ and $\Gamma=45_{-13-13}^{+18+30} \mathrm{MeV}$ with the signal significance of $6.5 \sigma$. However, BaBar reported that they didn't see a significant $\mathrm{Z}$ signal $(1.9 \sigma)$.

The observation of $Z^{+}(4430)$ motivated searches for other charged charmoniumlike states such as $\bar{B}^{0} \rightarrow K^{-} \pi^{+} \chi_{c 1}$ decays [6]. The signal appears as an horizontal band of entries in the Dalitz plot. We formulated a fitting function for the full Dalitz plot that assumes two-body decays of a superposition of various Breit Wigner function amplitudes that includes all known $K^{-} \pi^{+}$ resonances and $\pi^{+} \chi_{c 1}$ resonances. If we add two $\pi \chi_{c 1}$ resonances denoted by $Z_{1}$ and $Z_{2}$ in the fit, the fit result is favored over one resonance assumption at the $5.7 \sigma$ and no $Z$ assumption at more than $10 \sigma$ level. Figure 3 shows the projected $\pi^{+} \chi_{c 1}$ mass distribution for the mass range of 1.0 $<M^{2}\left(K^{-} \pi^{+}\right)<1.75 \mathrm{GeV}^{2}$ in the Dalitz plot, which is the most sensitive to $Z_{1}$ and $Z_{2}$.

We applied the same Dalitz Plot formalism as used in $B \rightarrow K \pi \chi_{c 1}$ to $B \rightarrow K \pi \psi^{\prime}$ [7]. The same intermediate $K \pi$ resonances are included in amplitudes in the fit model except for the $K_{3}^{*}(1780)$ which is well above the $K \pi$ phase space boundary in $B \rightarrow K \pi \psi^{\prime}$ decays. Figure 4 A) shows $M^{2}\left(\pi \psi^{\prime}\right)$ projections from corresponding vertical slices in Dalitz plot. Common peaks appear from slices (a), (c) and (e), while the signal is not clear in (b) and (d). The fit model assuming a $\pi^{+} \psi^{\prime}(Z)$ resonance and all known intermediate $K \pi$ resonances gives a fit confidence level $36 \%$. The statistical significance is $6.4 \sigma$. If we assume no $\pi^{+} \psi^{\prime}(Z)$ resonance, all known $K \pi$ resonances cannot reproduce the peak and the fit confidence level of $0.1 \%$ is poor, too. The fit result gives a mass and width of $\mathrm{M}=4443_{-12-13}^{+15+17} \mathrm{MeV}, \Gamma_{Z}=109_{-43-52}^{+86+57} \mathrm{MeV}$, and a product branching fraction $B\left(\bar{B}^{0} \rightarrow K^{-} Z^{+}(4430)\right) \times B\left(Z^{+} \rightarrow \pi^{+} \psi^{\prime}\right)=\left(3.2_{-0.9-1.6}^{+1.8+5.3}\right) \times 10^{-5}$. The mass and significance are similar to those in the previous measurement, but the width and its errors are larger. The branching fraction is comparable to those of other charmoniumlike states including the $Z_{1}^{+}$and $Z_{2}^{+}$.

\section{The $X(3872)$, and the $1^{--} Y$ states}

The $X(3872)$ was first observed in $J / \psi \pi^{+} \pi^{-}$mass in $B \rightarrow K X(3872)$ decays [8]. The world averged value of its mass in $J / \psi \pi^{+} \pi^{-}$channel only is $M_{X(3872)}=3871.5 \pm 0.2 \mathrm{MeV}$ when include the updated mass by Belle [9] and by CDF [10], while the threshold mass of $D^{0} D^{* 0}$ system is $3871.8 \pm 0.4 \mathrm{MeV}$ [11]. This similarity in mass is one important feature of the $X(3872)$, from 

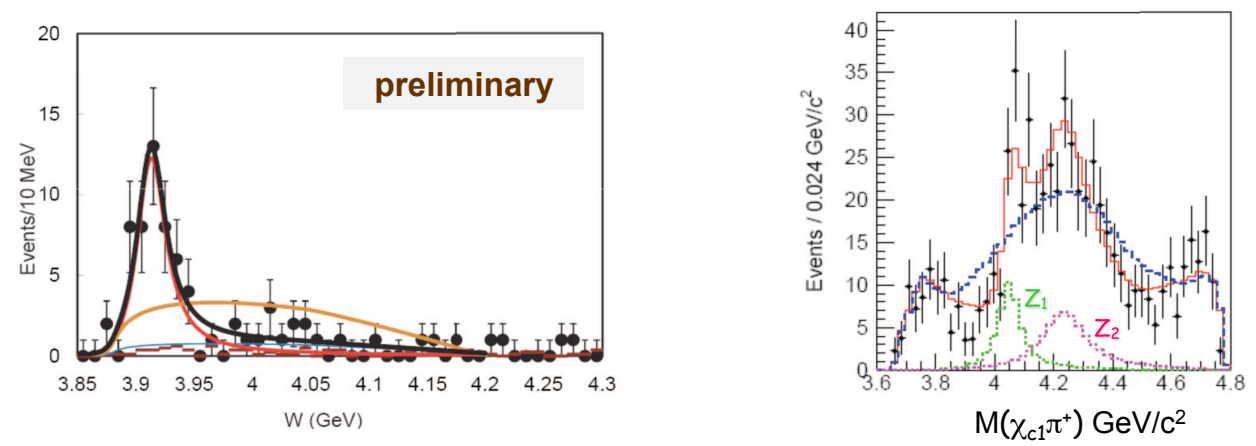

Figure 2: Left panel: The $W$ distribution for candidate events of $\gamma \gamma \rightarrow \omega J / \psi$. The black, red and blue curves show the total, resonance and background, respectively. The orange color curve shows the fit with no resonance assumption.

Figure 3: Right panels: The $M\left(\pi^{+} \chi_{c 1}\right)$ distribution in the range of $1.0 \mathrm{GeV}^{2}<M^{2}\left(K^{-} \pi^{+}\right)<1.75 \mathrm{GeV}^{2}$, which is most sensitive to the $Z_{1}$ and $Z_{2}$. The dots with error bars represent data, the solid (dashed) histogram is the Dalitz plot fit model with all known $K^{*}$ and two (none) $\pi^{+} \chi_{c 1}$ resonances.

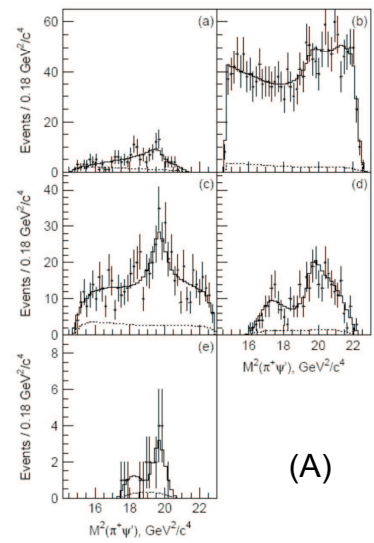

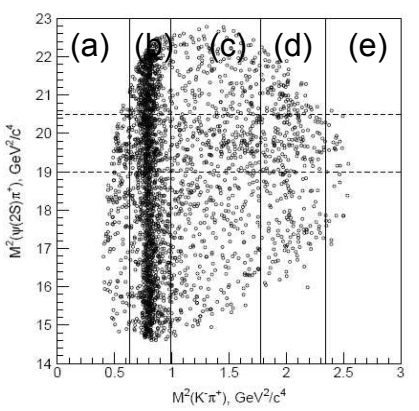

(B)

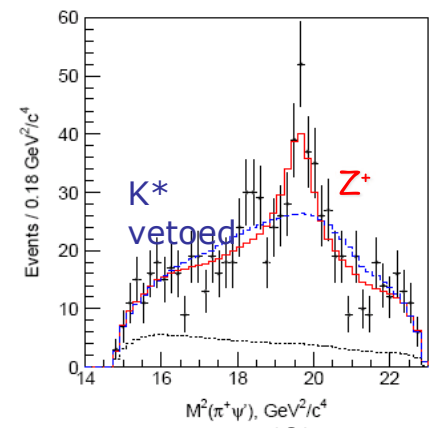

(C)

Figure 4: A) The $M^{2}\left(\pi^{+} \psi^{\prime}\right)$ distribution projected from the vertical slices (a) - (e) in the Dalitz plot B). B) $M^{2}(\pi \psi \prime)$ vs $M^{2}(K \pi) \mathrm{GeV}^{2}$ for the $B \rightarrow K \pi^{+} \psi \prime$ candidate. C) The $M^{2}\left(\pi^{+} \psi^{\prime}\right)$ distribution projected from vertical slices (a),(c) and (e) in the Dalitz plot B). The dots with error bars represent data, the red (blue) histogram is the Dalitz plot fit model with a $\pi^{+} \psi^{\prime}$ (none) resonance and all known $K \pi$ resonances.

which the $D \bar{D}^{*}$ molecule model was predicted. This motivated the search for the $X(3872) \rightarrow D \bar{D}^{*}$ decays. Both Belle and BaBar group measured a higher mass of $3875.2 \pm 0.7_{-1.6}^{+0.3} \pm 0.8 \mathrm{MeV}$ and $3875.1_{-0.5}^{+0.7} \pm 0.5 \mathrm{MeV}$. The updated mass in this channel [12] with 1.5 times bigger data sample is $3872.6_{-0.4}^{+0.5} \pm 0.4 \mathrm{MeV}$, by requiring $D^{*}$ decaying to either $D^{0} \gamma$ or $D^{0} \pi^{0}$. This is consistent with the world averaged value determined from the $\pi^{+} \pi^{-} J / \psi$ mode and shows no sign of a mass doublet predicted by diquark-diantiquark model. In addition, Braaten et al. have pointed out that the mass and width measured in the $D \bar{D}^{*}$ final state do not exactly match those of the $X(3872)$ resonance [13].

The $1^{--} \mathrm{Y}(4260)(\mathrm{Y}(4325))$ state first seen in $J / \psi \pi^{+} \pi^{-}\left(\psi^{\prime} \pi^{+} \pi^{-}\right)$masses were discovered in $e^{+} e^{-} \rightarrow \gamma_{I S R} Y$ by BaBar. Belle confirmed both states in the same decay modes. An interesting 
question is whether there exists any $X Y Z$ counter parts in the $s \bar{s}(b \bar{b})$ system. The candidate $Y_{s}$ state via initial state radiation in $s \bar{s}$ sector was first reported by $\mathrm{BaBar}$ in the $f_{0} \phi$ production cross section in $e^{+} e^{-} \rightarrow \gamma_{I S R} Y_{S}$ and confirmed by the BES. We also measured the $\phi f_{o}$ cross sections produced in $e^{+} e^{-} \rightarrow \gamma_{I S R} \phi f_{0}[14]$.

We measured $e^{+} e^{-} \rightarrow \Upsilon(n S) \pi \pi$ production cross section at seven different mass points near by the $\Upsilon(5 S)$ resonance at $\sqrt{s} \sim 10.87 \mathrm{GeV}$ and observed a new peak structure that a BW fit gives a mass and width of $\mathrm{M}=10889.6 \pm 1.8 \pm 1.5 \mathrm{MeV}$ and $\Gamma=54.7_{-7.2}^{+8.5} \pm 2.5 \mathrm{MeV}$ [15]. These parameters are not consistent with any known $b \bar{b}$ state such as $\Upsilon(10860)$ and the $\pi \pi \Upsilon(n S)$ cross section exceeds by more than 2 order of magnitude that for any conventional $b \bar{b}$ state. This can be interpreted as a candidate of $Y_{b}$ state in $b \bar{b}$ system.

\section{Summary}

We presented the first observation of a new charmoniumlike enhancement in $\gamma \gamma \rightarrow \omega J / \psi$ process. The $Z^{+}(4430)$ in $\pi^{+} \psi^{\prime}$ mass has been reanalyzed using a Dalitz plot formalism that includes all possible intermediate $K \pi$ resonances. As a result, the $Z^{+}(4430)$ signal is confirmed and mass and width are updated. The updated mass of $X(3872)$ and the candidate of $Y$ type state in $b \bar{b}(s \bar{s})$ are also briefly mentioned.

\section{References}

[1] P. Pakhlov et al. (Belle Collaboration), Phys. Rev. Lett. 100, 202001 (2008).

[2] S.-K. Choi, S. L. Olsen et al. (Belle Collaboration), Phys. Rev. Lett. 94, 182002 (2005).

[3] S. Uehara et al. (Belle Collaboration), Phys. Rev. Lett. 96, 082003 (2006).

[4] S. Uehara et al. (Belle Collaboration), in preparation.

[5] S.-K. Choi, S. L. Olsen et al. (Belle Collaboration), Phys. Rev. Lett. 100, 14200 (2008).

[6] R. Mizuk, R. Chistov et al. (Belle Collaboration), Phys. Rev. D78, 072004 (2008).

[7] R. Mizuk et al. (Belle Collaboration), Phys. Rev. D80 031104 (2009).

[8] S.-K. Choi, S. L. Olsen et al. (Belle Collaboration), Phys. Rev. Lett. 91, 262001 (2003).

[9] I. Adachi et al. (Belle Collaboration), arXiv:0809.1224 (2008).

[10] A. Abulencia et al. (CDF Collaboration), arXiv:0906.5218 (2009).

[11] C. Amsler et al. (Particle Data Group), Phys. Lett.B667 (2008).

[12] I. Adachi et al. (Belle Collaboration), arXiv:0810.0358 (2008).

[13] E. Braaten and J. Stapleton, arXiv:0907.3167 (2009).

[14] C. P. Shen et al. (Belle Collaboration), arXiv:0808.0006 (2008).

[15] K.F.Chen et al. (Belle Collaboration), Phys. Rev. Lett. 100, 112001 (2008). 